\title{
How to Make Fragile Bonds No Longer Fragile towards Electrons for Robust Organic Optoelectronic Materials
}

\author{
Rui Wang, ${ }^{[a]}$ Qing-Yu Meng, ${ }^{[a]}$ Yi-Lei Wang, ${ }^{[a]}$ and Juan Qiao*[a,b] \\ [a] R. Wang, Q.-Y. Meng, Dr. Y.-L. Wang, Dr. J. Qiao \\ Key Lab of Organic Optoelectronics and Molecular Engineering of Ministry of Education, Department of Chemistry, Tsinghua University, \\ Shuangqing Road 30, Haidian District, Beijing 100084 \\ Beijing ( $P$. R. China) \\ E-mail: qjuan@mail.tsinghua.edu.cn \\ [b] Dr. J. Qiao \\ Center for Flexible Electronics Technology, Tsinghua University \\ Shuangqing Road 30, Haidian District, Beijing 100084 \\ Beijing (P. R. China) \\ Supporting information for this article is given via a link at the end of the document.
}

\begin{abstract}
The development of robust organic (opto)electronic devices is mainly depressed by the chemical deterioration of organic materials on service. For organic light-emitting diode (OLED) materials, a key parameter for intrinsic stability is the bond-dissociation energy of the most fragile bond $\left(\mathrm{BDE}_{\mathrm{f}}\right)$. Although less concerned, many OLED molecules have the lowest $\mathrm{BDE}_{\mathrm{f}}$ in anionic states $\left(\mathrm{BDE}_{\mathrm{f}}(-) \sim 1.6-2.5 \mathrm{eV}\right)$, which could be a fatal short-slab for device stability. Here, we confirmed the relationship between $\operatorname{BDE}_{f}(-)$, intrinsic material stability, and device lifetime. To obtain high $\operatorname{BDE}_{\mathrm{f}}(-)$, we uncovered that introducing strong electron-withdrawing groups with delocalizing structures is an effective and general strategy, which substantially improves $\operatorname{BDE}_{\mathrm{f}}(-)$ for various fragile bonds by $\sim 1 \mathrm{eV}$. This was verified in typical phosphine-oxide and carbazole molecules, and backed by newly-designed molecules. Importantly, this tactic provides a particular perspective to employ the vulnerable building blocks, which largely enriches alternative blocks for robust organic (opto)electronic materials.
\end{abstract}

\section{Introduction}

The operational stability is a crucial and common issue for organic (opto)electronic devices. ${ }^{[1]}$ In particular, it is one of the greatest remaining problems for the popular organic light-emitting diodes (OLEDs). To date, lifetime of efficient blue OLEDs (exciton energy usually $\geq 2.7 \mathrm{eV}$ ) still cannot satisfy practical applications. The intrinsic degradation of OLEDs is mainly ascribed to the chemical deterioration of organic (or metal-organic) materials. ${ }^{[1,2]}$ Many undesired (photo)physical processes could induce such chemical deterioration, like exciton-polaron and exciton-exciton annihilations (EPA and EEA), in which EPA has been confirmed as a dominate mechanism. ${ }^{[3]}$ In that process (Figure 1a), one exciton transfers energy to a polaron, generating an excited polaron whose energy could be high enough to break chemical bonds and incur chemical deteriorations. In past decades, considerable efforts have been made to suppress the undesired (photo)physical processes. ${ }^{[3 a, 4]}$ However, completely avoiding them at the microscopic level is scarcely possible, while even a small amount of deterioration products can result in $50 \%$ luminance loss. ${ }^{[3 a, 5]}$ Therefore, restraining the induced (photo)chemical deteriorations is essential.

According to thermodynamics, the bond most probable to break would be the fragile bond with the minimum (or comparable-to-theminimum) bond-dissociation energy $(B D E)$ in that molecule. Its $B D E\left(B D E_{f}\right)$ has been confirmed as a key parameter for the intrinsic stability of OLED materials by mounting evidences. ${ }^{[6]}$ In general, the chemical bonds of organic molecules are particularly vulnerable in anionic states. In Figure $1 \mathrm{~b}$ and $1 \mathrm{c}$, we listed the $\operatorname{BDE}(\mathrm{n}), \operatorname{BDE}(+)$, and $\operatorname{BDE}(-)(n,+$, and - refer to neutral, cationic and anionic states) values of typical fragile exocyclic $C-X$ single bonds $(X=$ heteroatoms like $N, P, S$, etc.) in several representative OLED molecules. It can be found that the $\mathrm{BDE}_{\mathrm{f}}(\mathrm{n})$ and $\mathrm{BDE}_{\mathrm{f}}(+)$ of most interested molecules are 3.1-4.8 eV, while most $\mathrm{BDE}_{\mathrm{f}}(-)$ are only 1.6-2.5 eV. Many OLED molecules have comparable $B D E_{f}$ values. ${ }^{[2 b, 6]}$ As a result, once such negative polarons are generated and/or get involved in EPA, the fragile bonds would dissociate and incur chemical deterioration. Therefore, $\operatorname{BDE}_{\mathrm{f}}(-)$ would be a fatal short-slab of the intrinsic stability for OLED materials, and deserves a special focus in molecular stability and the related device degradation study.

The intrinsic degradation of OLED materials towards electrons was first reported by Aziz et al. in tris(8-hydroxyquinoline) aluminum $\left(\mathrm{Alq}_{3}\right)$-based devices. ${ }^{[7]}$ They found that the residence of excessive electrons in $\mathrm{Alq}_{3}$ layer would incur the luminescence efficiency loss of the device. It is assumed that electron traps of unknown nature (chemical degradation is suggested) with a luminance efficiency of are formed. For OLED materials, we first concerned BDE values of charged states in the study of phosphine-oxide materials, ${ }^{[6 \mathrm{~d}]}$ and found that the typical host $\mathrm{CzPO} 2$, whose $\mathrm{BDE}_{f}(-)$ is only $2.15 \mathrm{eV}$, showed serious $\mathrm{C}-\mathrm{P}$ cleavage in electron-only devices. Since then, a cognition has been accepted that $\mathrm{PO}$ could undermine the device stability due to the fragile $\mathrm{C}-\mathrm{P}$ bond. ${ }^{[2 \mathrm{~b}, 6 \mathrm{ee}, 6 \mathrm{k}, 8]}$ Later, Lee et al. found the carbazole $(\mathrm{Cz})$-based host with higher BDE in charged states contributed to the longer device lifetime. ${ }^{\text {[9a] }}$ Recently, more explores have demonstrated prolonged the device lifetime based on the consideration of $\operatorname{BDE}(-) .{ }^{[9]}$ To date, studies on the regulation of $\operatorname{BDE}(-)$ for organics are mainly about organic halides. ${ }^{[10]}$ For OLED molecules, Brédas et al. ${ }^{[6]]}$ recently looked into the substituent effects of the cyano, fluorine, and hydroxyl groups on the BDE values of the $\mathrm{C}-\mathrm{N}$ bonds in the typical hosts $\mathrm{Cz}$-dibenzothiophene (DBT) derivatives, and found that the $\mathrm{BDE}(-)$ could be improved by increasing the relative electron affinity of the DBT moiety. However, it still remains largely elusive about the rational regulation of $\mathrm{BDE}_{\mathrm{f}}(-)$ for various OLED molecules. 
Different from $\operatorname{BDE}_{f}(n)$ which mainly depends on the bond type, $\operatorname{BDE}_{f}(-)$ is largely affected by the whole molecular structure, as evidenced by the almost identical $\mathrm{BDE}_{\mathrm{f}}(\mathrm{n})$ and very different $\mathrm{BDE}_{\mathrm{f}}(-)$ of the same $\mathrm{C}-\mathrm{N}$ or $\mathrm{C}-\mathrm{P}$ bonds in molecules 4-7 (Figure 1c). For example, although ptBCZPO2TPTZ has the same $\mathrm{C}-\mathrm{P}$ bond as $\mathrm{CzPO} 2$ does, its $\mathrm{BDE}_{\mathrm{f}}(-)$ is as high as $3.15 \mathrm{eV}, 1 \mathrm{eV}$ higher than that of CzPO2. We found it did not show observable deterioration in laser-desorption/ionization time-of-flight mass spectrometry (LDI-TOFMS) until very high laser powers (Figure S1), suggesting its C-P bonds are relatively stable, although the authors did not consider the stability issues. ${ }^{[11 a]}$ This finding indicates that the original fragile bonds can be turned into stable ones, which is rarely recognized in the previous development of OLED materials, and it drove us to explore the general effective strategy to manage $\operatorname{BDE}_{\mathrm{f}}(-)$, which will make an essential contribution to improving the operational stability of OLEDs and other organic (opto)electronic devices.

Herein, we first conducted comprehensive experiments and theoretical calculations, and revealed how $\operatorname{BDE}_{f}(-)$ affects intrinsic material stability and device lifetime. Considering that the PO group is known to have $\operatorname{BDE}_{f}(-)$ issues, but are still one of the most popular electron acceptors in OLED materials (especially in high-efficiency hosts), ${ }^{[11]}$ PO-derivatives would be meaningful representatives for studying $\operatorname{BDE}_{\mathrm{f}}(-)$. In addition, since $\mathrm{Cz}$ is nearly the most common electron donor in OLED molecules, ${ }^{[2]}$ and the corresponding $\mathrm{C}-\mathrm{N}$ bonds have demonstrated $\mathrm{BDE}_{\mathrm{f}}(-)$ issues in some cases, ${ }^{[6]} \mathrm{Cz}$ derivatives also deserves a thorough study. We thus took a systematic theoretical study on typical $\mathrm{PO}$ and $\mathrm{Cz}$-derivatives, and developed an effective and general strategy to rationally manage $\operatorname{BDE}_{f}(-)$ according to fundamental thermodynamics, which was validated by the comparisons in several groups of reported and newly designed molecules. Importantly, since this tactic can significantly improve $\operatorname{BDE}_{\mathrm{f}}(-)$ (often by $\sim 1 \mathrm{eV}$ ), it actually provides a new perspective for reviving the originally vulnerable building blocks (like PO) instead of being casted away. Thus, this tactic can largely enrich the alternative blocks for the development of robust OLED and other organic (opto)electronic materials.

(a)

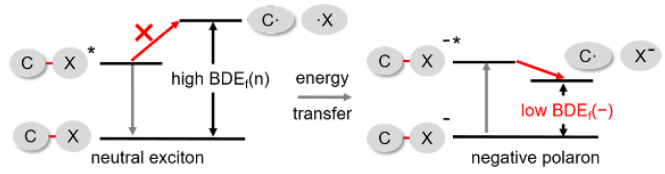

(b)
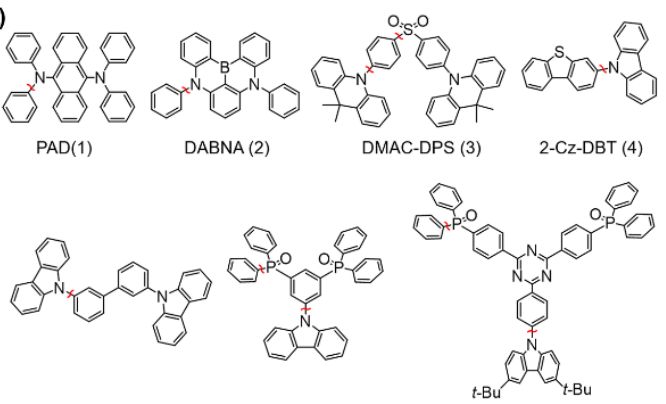

$\operatorname{mCBP}(5)$

$\mathrm{CzPO} 2(6)$

ptBCzPO2TPTZ (7)

(c)

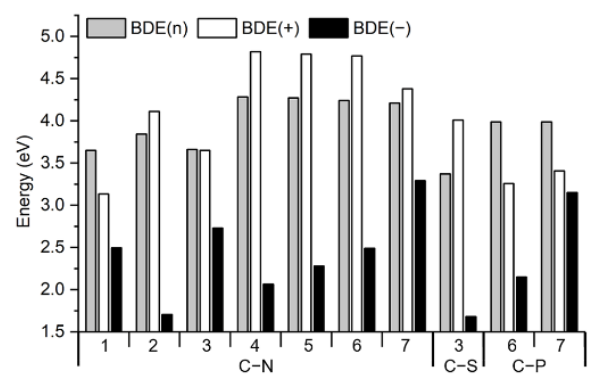

Figure 1. a) Schematic of the potential mechanism of EPA-induced bond-dissociation of the negative polaron. The asterisk refers to the excited state. BDEf(n) and $\mathrm{BDE}_{\mathrm{f}}(-)$ refers to the bond-dissociation energies of the fragile $\mathrm{C}-\mathrm{X}$ bonds under neutral and anionic states, respectively. b) Chemical structures of typical OLED molecules 1-7. The fragile bonds are highlighted by the red markers. c) BDE $\mathrm{f}_{f}$ values of the interested molecules. All calculations were at the M06-2X/def2-SVP level.

\section{Results and Discussion}

\section{Comparative Studies on TP3PO and PO-T2T}

To separate the concerned $\mathrm{BDE}_{\mathrm{f}}(-)$ from other material-related parameters (e.g. exciton energy and thermal stability), we comparatively studied the intrinsic stability of two representative PO-based electron-transporting materials (ETM), TP3PO and PO-T2T, which have very similar chemical structures (Figure 2a). ${ }^{[11, \mathrm{e}]}$ The structural similarity leads to many similar molecular parameters (Figure S2 and Table S1). As for molecular stability, $\mathrm{BDE}_{\mathrm{f}}(\mathrm{n})$ values in TP3PO and PO-T2T are all close to $4.00 \mathrm{eV}$ for the C-P bonds (details are in Table S2-S3), while their $\mathrm{BDE}_{\mathrm{f}}(-)$ values show large disparity, as low as 2.49 and $2.78 \mathrm{eV}$ for $\mathrm{C} 1-\mathrm{P}$ and C2-P bonds of TP3PO, but as high as 3.43 and $3.60 \mathrm{eV}$ for those of PO-T2T. The low BDE $\mathrm{f}_{\mathrm{f}}(-)$ of TP3PO would cause undesired chemical degradations, while the 
high $\operatorname{BDE}_{\mathrm{f}}(-)$ of PO-T2T is enough to afford exciton energies in most OLEDs, which may disburden this material of undesired degradations.

To validate these speculations, we conducted LDI-TOF-MS tests, which have proved to be powerful to study the degradations of OLED materials. ${ }^{[5,6 \mathrm{c}-\mathrm{e}]}$ Here, samples were the pure powder of TP3PO and PO-T2T. Under the negative detection mode, the laser intensity was set to increase gradually from 90 to $110 \mu \mathrm{J} /$ pulse to track the degradation process (all spectra are in Figure S3). At the lowest intensity of $90 \mu \mathrm{J} /$ pulse, TP3PO showed weak molecular and quasi-molecular ion peaks ([M $\pm \mathrm{H}]^{-}$, etc.), and C2-P bond cleavage peaks [M-POPh$]^{-}$; but very strong $\mathrm{C} 1-\mathrm{P}$ bond cleavage peaks $[\mathrm{M}-\mathrm{Ph}]^{-}$and $[\mathrm{M}[\mathrm{O}]-\mathrm{Ph}]^{-}$. As the laser intensity increased, the $\left[\mathrm{M} \pm \mathrm{H}^{-}\right.$and $\left[\mathrm{M}-\mathrm{POPh}_{2}\right]^{-}$almost disappeared, while the $[\mathrm{M}-\mathrm{Ph}]^{-}$and $[\mathrm{M}[\mathrm{O}]-\mathrm{Ph}]^{-}$became very conspicuous. In comparison, at $90 \mu \mathrm{J} /$ pulse, PO-T2T showed strong $\left[\mathrm{M} \pm \mathrm{H}^{-}\right.$, but very weak $[\mathrm{M}-\mathrm{Ph}]^{-}$and $[\mathrm{M}[\mathrm{O}]-\mathrm{Ph}]^{-}$. It is not until the intensity exceeded 100 $\mu \mathrm{J} /$ pulse that the $[\mathrm{M}-\mathrm{Ph}]^{-}$and $[\mathrm{M}[\mathrm{O}]-\mathrm{Ph}]^{-}$became stronger than the $[\mathrm{M} \pm \mathrm{H}]^{-}$. The $\left[\mathrm{M}-\mathrm{POPh}_{2}\right]^{-}$of $\mathrm{PO}-\mathrm{T} 2 \mathrm{~T}$ was very weak throughout the entire process.

The LDI-TOF-MS results accord well with BDE predictions. Specifically, (i) For both TP3PO and PO-T2T, C1-P bonds with lower $\mathrm{BDE}$ are more fragile than $\mathrm{C} 2-\mathrm{P}$ bonds. (ii) TP3PO with lower $\mathrm{BDE}_{\mathrm{f}}(-)$ showed poorer material stability. These results strongly support that $\mathrm{BDE}_{f}$ is a key molecular parameter for the intrinsic material stability. Since QC-calculations and experiments both suggest that $\mathrm{C} 1-\mathrm{P}$ ( $\mathrm{C} 1$ means the unsubstituted phenyl) are more fragile than $\mathrm{C} 2-\mathrm{P}$ bonds, the following studies on $\mathrm{PO}$-derivatives mainly focus on C1-P bonds.

\section{Device degradation Experiments}

To disclose the material stability with device lifetime, we first fabricated TP3PO- and PO-T2T-based electron-only devices, with the structure ITO|PO-T2T $(120-x \mathrm{~nm}) \mid$ TP3PO $(x \mathrm{~nm})|\mathrm{TPBi}(20 \mathrm{~nm})| \mathrm{LiF}(1 \mathrm{~nm}) \mid \mathrm{Al}(120 \mathrm{~nm})$ (Figure 2b). Under the electrical stress (10 mA $\mathrm{cm}^{-2}$ ), we found that the aging of the EODs become more rapid as the thickness of TP3PO increases. For $x=0,5,20$, and 120, the voltage increases $(\Delta V)$ of the EODs after $5 \mathrm{~h}$ stress are $0.04,1.57,4.41$, and $20.52 \mathrm{~V}$, respectively (Figure $2 \mathrm{c}$ ). This result clearly demonstrates that the instability of TP3PO anion is the main cause of the voltage rise. Next, we fabricated OLEDs with the structure ITO|HATCN $(10 \mathrm{~nm}) \mid$ NPB $(30 \mathrm{~nm}) \mid$ TCTA $(15 \mathrm{~nm}) \mid$ mCBP $(15 \mathrm{~nm})$ |DPEPO:30 wt\% TCzTrz $(30 \mathrm{~nm}) \mid$ PO-T2T $(40-x \mathrm{~nm}) \mid$ TP3PO $(x$ $\mathrm{nm})|\mathrm{TPBi}(5 \mathrm{~nm})| \mathrm{LiF}(1 \mathrm{~nm}) \mid \mathrm{Al}(120 \mathrm{~nm})\left(x=5,10,30\right.$, or 35; Figure 2d). TCzTrz is a sky-blue emitter developed by Zhang et al. ${ }^{[12]}$ All these OLEDs demonstrated close maximum efficiencies ( 11\%, Figure S5), which are comparable to reported values. Figure $2 \mathrm{e}$ shows their half-life (LT50) at an initial brightness of $500 \mathrm{~cd} \mathrm{~m}^{-2}$. For the devices $x=5,10,30$, and 35, LT50 values are 12.0, 11.0, 7.7, and $4.2 \mathrm{~h}$, respectively (Figure 2e). Since the only difference between these devices is the thicknesses of TP3PO and PO-T2T, the result further supports that it is the TP3PO with much lower $\mathrm{BDE}_{\mathrm{f}}(-)$ that accounts for device degradations.

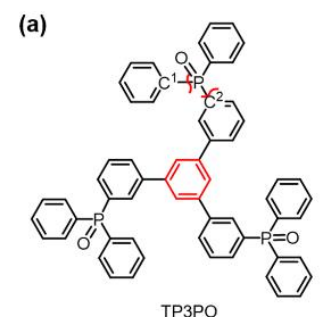

(b)

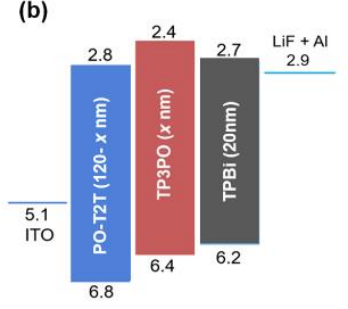

(d)

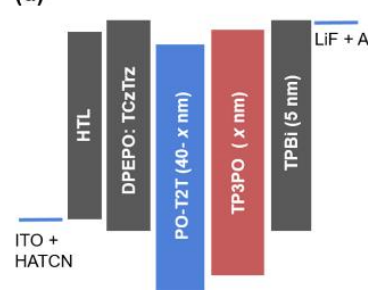

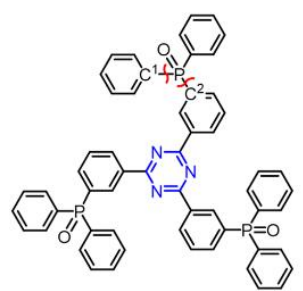

PO-T2T

(c)

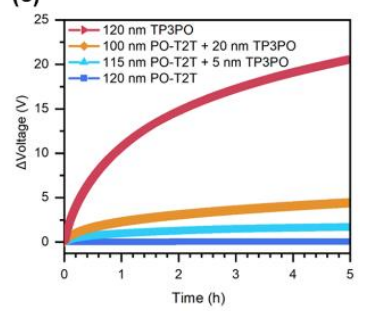

(e)

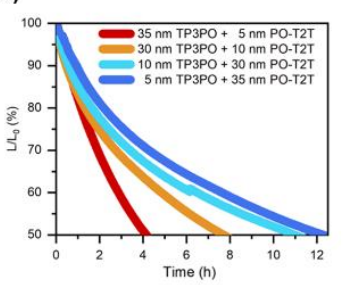

Figure 2. a) Chemical structures of TP3PO and PO-T2T, the fragile bonds are highlighted by the red markers. b) Device structures of the EODs ( $x=0,5,20$, or 120). The current density-voltage curves of the devices are in Figure S4. c) Change of the voltages of EODs during $5 \mathrm{~h}$ under a constant current density of $10 \mathrm{~mA}$ $\mathrm{cm}-2$. d) Device structures of the OLEDs $(x=5,10,30$, or 35$)$. Chemical structures of the involved organic materials are shown in Figure S5. To prevent the introduction of new interfaces, devices with solely TP3PO (or PO-T2T) as the ETM were not fabricated. e) The operation lifetime of the OLEDs measured at a brightness of $500 \mathrm{~cd} \mathrm{~m}-2$ under a constant current. 
To rationally link the macroscopic device degradation with the microscopic bond cleavage, we further calculated energy levels of POcontaining anions resulted from the bond cleavage of TP3PO and PO-T2T. Figure S6 shows that HOMOs of PO-containing anions $(-1.72$ to $-2.20 \mathrm{eV})$ are much lower than LUMOs $(-0.79$ to $-1.27 \mathrm{eV})$ of the intact molecules. Hence, once generated, these anions would act as filled-deep traps, hindering the electron transport and injection. Since TP3PO is more fragile towards electrons, the corresponding devices will generate much more defects in the same time scale, thus showing poorer device stability.

Up to here, we comprehensively demonstrated the close relationship between $\operatorname{BDE}_{f}(-)$, intrinsic material stability, and device lifetime, and revealed that active organic materials with lower $\mathrm{BDE}_{\mathrm{f}}(-)$ would result in poorer material stability and device lifetime. It is noteworthy that the comparison between TP3PO and PO-T2T demonstrates that with appropriate molecular design, the original vulnerable groups like $\mathrm{PO}$ can serve in robust materials, although that is rarely recognized before. Therefore, it would be imminent to reveal the relationship between $\operatorname{BDE}_{f}(-)$ and molecular structure, and establish feasible strategies to improve $\operatorname{BDE}_{f}(-)$.

\section{Key Influence Factors of $\operatorname{BDE}_{\mathrm{f}}(-)$}

For the $\mathrm{C}-\mathrm{X}(\mathrm{X}=\mathrm{N}, \mathrm{P}, \mathrm{S}$, etc.) bonds in OLED molecules, $\mathrm{X}$-sides usually have higher electronegativities, so the bond dissociations in anionic states will result in anions containing $X$. According to the Hess's law, an equation between $\operatorname{BDE}_{f}(-)$ and $B E_{f}(n) \operatorname{can}_{\text {be derived }}$ (Scheme 1),

$$
\operatorname{BDE}_{f}(-)=\mathrm{BDE}_{\mathrm{f}}(\mathrm{n})+\mathrm{EA}_{\mathrm{M}}-\mathrm{EAx}
$$

Scheme 1. Derivation of the calculation formula of $\operatorname{BDE}_{\mathrm{f}}(-)$.

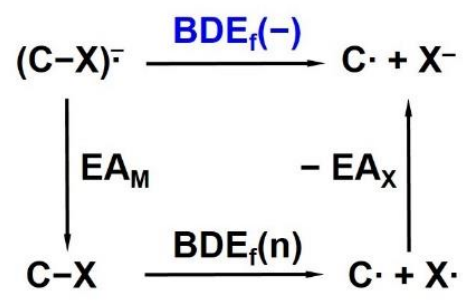

where $E A_{X}$ and $E A_{M}$ represent the electron affinity of the $X$ radical and the intact molecule, respectively. For all $C-X$ bonds in Figure $1 b$, it is their $E A_{x}$ values $(2-2.6 \mathrm{eV})$ significantly higher than $E A_{M}$ values $(0.3-1.5 \mathrm{eV})$ that lead to largely reduced $B D E_{f}(-)$. Most importantly, the equation suggests how to control $\operatorname{BDE}_{f}(-)$. For $\operatorname{BDE}_{f}(n)$, it mainly depends on the type of $C-X$ bond and rarely influenced by other parts of the molecule except ortho-substituents. ${ }^{26,34}$ Although ortho-substituents may cause large influences (up to $0.5 \mathrm{eV}$ ), the effects depend on case-specific spatial factors and electronic characters of the substituents. ${ }^{26}$ So the ortho-effects are not involved here. For $E A_{M}$, it can be ideally increased by introducing electron-withdrawing groups (EWGs) on C-side of C-X bond. It is feasible when $\mathrm{X}$ is an atom (e.g. halogen). However, in OLED molecules, $\mathrm{X}$-sides are usually building blocks. Moreover, many OLED molecules have several $\mathrm{C}-\mathrm{X}$ bonds (Figure $1 \mathrm{~b}$ ), modifications on the $\mathrm{C}$-side of one $\mathrm{C}-\mathrm{X}$ bond may be right on the $\mathrm{X}$-sides of the others. In these cases, introducing $E W G$ s would lead to simultaneous increase of $E A_{M}$ and $E A_{X}$, which may result in a limited increase or even decrease of $\mathrm{BDE}_{f}(-)$. The question then arises: to obtain a high $\mathrm{BDE}_{\mathrm{f}}(-)$ for a given $\mathrm{C}-\mathrm{X}$ bond, how to improve $E A_{M}$ accurately without increasing $E A_{X}$ as much as possible? To address this, an in-depth study between $E A_{M}, E A_{X}$ and molecular structure is indispensable.

\section{Improving $\operatorname{BDE}_{\mathfrak{f}}(-)$ Values of PO-derivatives}

We first looked into the parent molecule $\mathrm{POPh}_{3}(\mathbf{P} 1)$. Figure 3 a shows the negative charge in $\mathrm{POPh}_{3}$ anion mainly distributes on its delocalized LUMO, while that of the $\mathrm{POPh}_{2}$ fragment anion mainly locates on sp3-orbital of $P$. Thus, $E A_{M}$ and $E A_{x}$ are basically determined by the distinct orbitals. To increase $E A_{M}$, introducing strong EWGs or delocalizing structures are two typical ways. Meanwhile, they would have smaller impact on $\mathrm{EA}_{\mathbf{x}}$ due to the localization of the sp ${ }^{3}$-orbital. Accordingly, we designed P2-P4 with strong EWGs, including trifluoromethyl (P2), cyano (P3), or replace one of the phenyl with 1,3,5-triazine (P4); and P5-P6 with relatively weaker EWGs but delocalizing structures, including pyridine (P5) and [1,1':3',1"-terphenyl]-5'-yl (PTP) (P6) (Figure 3b). Calculations show that $E A_{M}$ values of P2-P6 all increase by $0.6-1 \mathrm{eV}$, higher than the increase of $E A_{x}$ values $(\leq 0.45 \mathrm{eV})$. Of note, EAx values of $\mathbf{P 5}-\mathbf{P 6}$ are $\sim 0.2 \mathrm{eV}$ smaller than those of $\mathbf{P 2}-\mathbf{P 4}$. In total, $\mathrm{BDE}_{\mathrm{f}}(-)$ values of $\mathbf{P 2}-\mathbf{P 6}$ increase to $2.15-2.55 \mathrm{eV}$, which are considerably higher than that of $\mathbf{P} \mathbf{1}(1.87 \mathrm{eV})$, yet still comparable to the $\mathrm{BDE}_{\mathrm{f}}(-)$ of the unstable TP3PO $(2.49 \mathrm{eV})$. Incidentally, substituents at metapositions of $P$ atom bring similar effects (Table S4). Thus, a greater enhancement is demanded.

Since strong EWGs and delocalizing structures can both increase $E A_{M}$, and the latter has a smaller influence on $E A_{x}$, we thus combined the two methods into a more effective strategy, namely introducing strong EWGs with delocalizing structures (D-EWGs), and designed $\mathbf{P 7}$ and $\mathbf{P 8}$. Indeed, introductions of benzonitrile in $\mathbf{P 7}$ and 4,6-diphenyl-1,3,5-triazin-2-yl (Trz) in P8 remarkably increase EA by over $1.2 \mathrm{eV}$. We found the contribution of the $\mathrm{POPh}_{2}$ moiety to LUMO is only $8.7 \%$ in $\mathbf{P 8}$, considerably smaller than those in $\mathbf{P 4}$ (24.7\%) and P6 (12.2\%), indicating that D-EWGs can effectively confine the negative charge, thus protecting the vulnerable parts. Moreover, D-EWGs could suppress the increase of EAx. For instance, P3 and P7 both have a cyano; while P7 has an increased distance between the cyano and $\mathrm{P}$ atom, thus leading to a smaller EAx increase (0.32 vs $0.45 \mathrm{eV}$ for P7 and P3). Consequently, DEWGs significantly improve BDE $_{f}(-)$ to 2.73 and $3.04 \mathrm{eV}$ for $\mathbf{P 7}$ and P8 (Figure $3 b$ ), which do outperform the effects of solely introducing of EWGs or delocalizing structures in P2-P6. Therefore, introducing D-EWGs could be an effective strategy for improving BDE $\mathrm{E}_{\mathrm{f}}(-)$. For aryl amide and sulfone derivatives, D-EWGs likewise increase their $\mathrm{BDE}_{\mathrm{f}}(-)$ values by $\sim 1 \mathrm{eV}$ (Table S5).

Importantly, D-EWGs not only improve the thermodynamic stability, as reflected by $\mathrm{BDE}_{\mathrm{f}}$, but suppress the kinetic process of bond cleavages. That is confirmed by the comparison between the rapid degradation of TP3PO-only EOD, and the robustness of PO-T2Tonly EOD. During C-P bond cleavages towards electrons, the electron on LUMO redistributes to $\mathrm{sp}^{3}$-orbital of $\mathrm{P}$. When there are D- 
EWGs, the redistribution will become more difficult. To visualize this, potential energy curves (PECs) and electrostatic potential (ESP) maps are plotted during C1-P bond cleavages in TP3PO and PO-T2T anions. Figure 3c shows that PECs of the two anions are almost identical when C-P distance is $\sim 1.8 \AA$. However, when the distance is over $2.6 \AA$, PECs of PO-T2T clearly increase faster than those of TP3PO. In ESP maps (Figure 3d), when C1-P distance is $1.8 \AA$, the negative ESP (red) is mainly allocated around the center for both molecules (also see Figure S7). While at $2.6 \AA$, the red color within the central of TP3PO becomes shallower; while that in POT2T scarcely changes. From $1.8 \AA$ to $2.6 \AA$, the negative charge allocated around the central aryl $\left(q_{\mathrm{Ar}}\right)$ changes from -0.76 to -0.60 for TP3PO, while in PO-T2T, $q_{\mathrm{Ar}}$ only changes from -0.75 to -0.72 . Finally, when the phenyl leaves, the negative charge is concentrated on PO, while $q_{\mathrm{Ar}}$ of PO-T2T is still higher than that of TP3PO ( $-0.35 v s-0.27$, Table S6). This result clearly reflects that Trz can effectively confine the negative charge. Accordingly, introducing D-EWGs is also a promising strategy to protect $\mathrm{C}-\mathrm{X}$ bonds and improve their kinetic stability towards electrons. Next, we further demonstrate that the strategy is also effective for $\mathrm{Cz}$-derivatives, even though $\mathrm{Cz}$ and $\mathrm{PO}$ have quite different electronic structures.

(a)

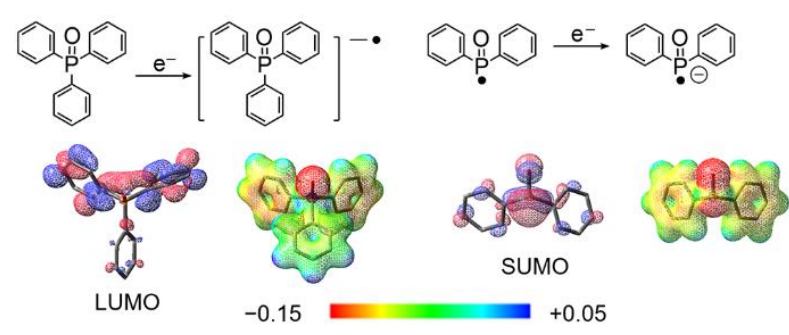

(b)

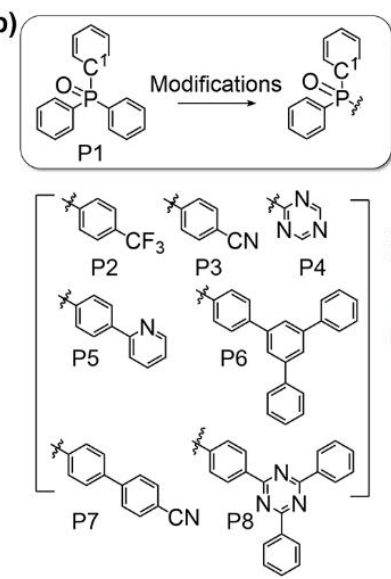

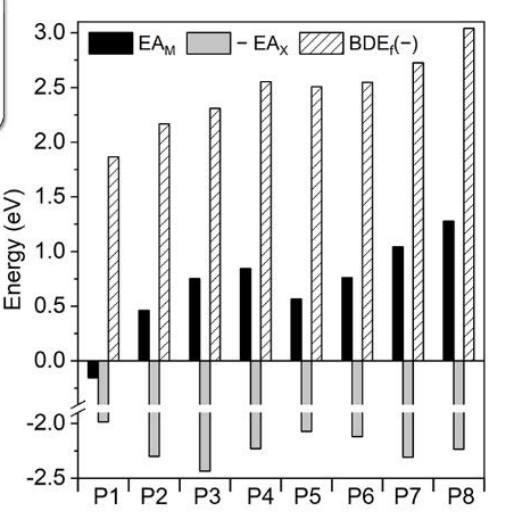

(c)

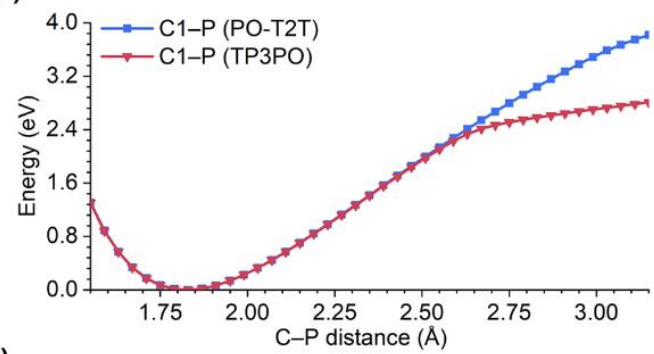

(d)

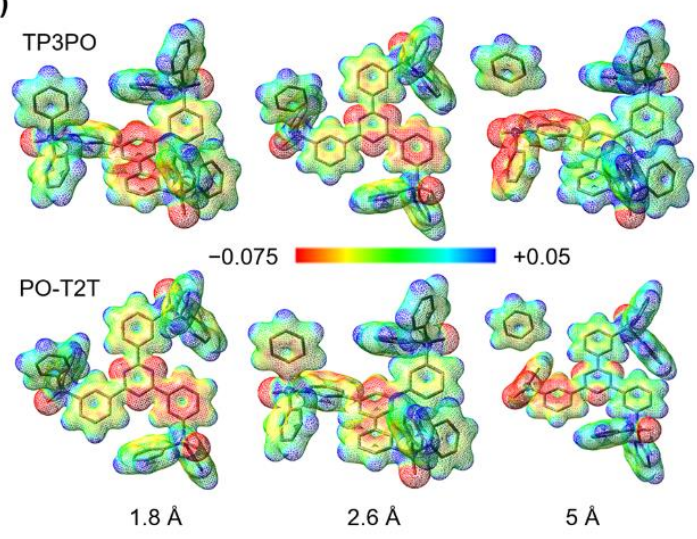

Figure 3. a) a) Frontier orbital (isovalue $=0.03 \mathrm{au}$ ) and $\mathrm{ESP}$ maps of $\mathrm{POPh} 3$ and $P O P h 2 . b)$ Chemical structures, $E A_{M}, E A_{x}$, and $B D E_{f}(-)$ values $($ with respect to C1-P bond) of molecules P1-P8. c) Potential energy curves with respect to the stretch of C1-P bonds (1.55-3.15 $\AA$ ) in TP3PO and PO-T2T anions. d) ESP maps (isovalue $=0.02 \mathrm{au}$ ) when the $\mathrm{C} 1-\mathrm{P}$ distance is $1.8,2.6$, and $5 \AA$, respectively. All calculations were at the M06-2X/def2-SVP level.

\section{Improving $\mathrm{BDE}_{\mathrm{f}}(-)$ Values of $\mathrm{Cz}$-derivatives}

For Cz-derivatives, substituents on $\mathrm{N}$-side or $\mathrm{C}$-side of the $\mathrm{C}-\mathrm{N}$ bond would have distinct effects on $E A_{M}$ and $E A_{x}$. We first studied the substituent effects on $\mathrm{N}$-side. Based on the parent PhCz (C1), we introduced the cyano, phenyl PTP, and phenyl Trz groups into 2 and 3-sites of $\mathrm{Cz}$, respectively. At 3-site, cyano dramatically increases $E A_{x}$ by $0.63 \mathrm{eV}$ due to its strong interaction with the delocalized $\mathrm{SUMO}$ of $\mathrm{Cz}$ radical (Figure $\mathrm{S} 8)^{[6]}$. Such increase is even higher than that of $E A_{M}(0.5 \mathrm{eV}$, Figure $4 \mathrm{~b})$, resulting in a decreased $B D E_{f}(-)$ (1.39 vs $1.45 \mathrm{eV}$ for $\mathbf{C} 2$ and $\mathbf{C} 1)$. In comparison, PTP brings much smaller increase of $E A_{X}(0.25 \mathrm{eV})$, but bigger increase of $E A_{M}(0.7$ $\mathrm{eV})$, thus leading to a higher $\mathrm{BDE}_{\mathrm{f}}(-)(1.89 \mathrm{eV})$ for C3. Likewise, Trz brings a smaller increase of $\mathrm{EA}_{\mathrm{x}}(0.36 \mathrm{eV})$, but much bigger increase of $\mathrm{EA}_{\mathrm{M}}(1.36 \mathrm{eV})$, thus leading to a significantly increased $\mathrm{BDE}_{\mathrm{f}}(-)(2.42 \mathrm{eV})$ for $\mathbf{C 4}$. Intriguingly, $\mathrm{BDE}_{\mathrm{f}}(-)$ values of the 2-sitesubstituted isomers are 1.78, 2.15, and $2.62 \mathrm{eV}$ for $\mathbf{C 5}, \mathbf{C 6}$, and C7, respectively; which are $0.2-0.4 \mathrm{eV}$ higher than those of 3-sitesubstituted isomers. That is because the substituent at 2-site has a stronger interaction with the LUMO of $\mathrm{Cz}$, but a relatively weaker interaction with the SUMO of $\mathrm{Cz}$ radical (Figure S9). Although 3,6-substitutions are widely used in $\mathrm{Cz}$-derivatives, the result here shows that 2,7-substitutions has greater advantage on improving the intrinsic molecular stability. In principle, substitutions on $\mathrm{C}$-side of PhCzderivatives solely increase $\mathrm{EA}_{\mathrm{M}}$ and thus improve $\mathrm{BDE}_{\mathrm{f}}(-)$. As shown in Figure 4b, cyano and Trz on the C-sides of C8 and C9 significantly increases their $\mathrm{BDE}_{\mathrm{f}}(-)$ to 2.26 and $3.02 \mathrm{eV}$, which are much higher than those of the $\mathrm{N}$-side-substituted counterparts.

\section{Improving $\operatorname{BDE}_{\mathrm{f}}(-)$ Values of Donor (D)- $\pi$-Acceptor (A) Molecules}

Most OLED molecules contain D-ח-A substructures, in which the fragile bonds are either in $D, A$ or in both. To explore the distinct effects of substituted position in D- $\mathrm{D}-\mathrm{A}$ molecules, we designed a group of molecules based on (3-(9H-carbazol-9$\mathrm{yl}$ )phenyl)diphenylphosphineoxide ( $\mathrm{CzPO}$, Figure $4 \mathrm{c})$, with $\mathrm{Cz}$ and $\mathrm{PO}$ as $\mathrm{D}$ and $\mathrm{A}$, respectively. Its $\mathrm{C}-\mathrm{P}$ and $\mathrm{C}-\mathrm{N}$ bonds have comparable BDE(-) values (1.90 vs $2.00 \mathrm{eV}$ ). The typical D-EWG Trz is introduced to the 2-site of $\mathrm{Cz}$ (D-Trz), para-position of POPh 2 (A-Trz), or on the central phenyl $(\pi-\mathrm{Trz})$. BDE $\mathrm{B}_{\mathrm{f}}(-)$ values in D-Trz, A-Trz, and $\pi$-Trz are 2.80, 2.89, and $3.08 \mathrm{eV}$ for $\mathrm{C}-\mathrm{P}$ bonds; and 
2.67, 3.26, and $3.20 \mathrm{eV}$ for $\mathrm{C}-\mathrm{N}$ bonds, respectively (Figure $4 \mathrm{~d}$ ). These values are 0.67-1.26 eV higher than those of CzPO. Compared with $D$ - or A-substituted counterparts, $\pi$-Trz simultaneously reinforces all the fragile bonds from $D$ and $A$. The framework not only makes each group contribute to $E A_{M}$, but also keeps them being spatially separated, preventing them from increasing $E A_{x}$ (the details are in Figure S10). Therefore, employing D-EWGs at the $\pi$-side (center) of the molecule is most recommended for developing robust $D-\pi-A$ molecules.

\section{Implications for Rational Design of Robust OLED Materials}

In OLED molecules, many building blocks can contribute to desirable optical/electrical performance. PO is a representative instance, which often plays an important role in the corresponding high-efficiency materials. ${ }^{[11]}$ Unfortunately, when PO is the sole acceptor, the corresponding molecules often suffer from low $\mathrm{BDE}_{\mathrm{f}}(-)$ values and poor material stability, as clearly demonstrated by $\mathrm{CzPO} 2$ and TP3PO. Similar issues would occur in A1-A3 as evidenced by the low $\mathrm{BDE}_{\mathrm{f}}(-)$ values $(1.48-2.33 \mathrm{eV}$, Figure $5 \mathrm{a}$ and $5 \mathrm{~b})$. To enhance the molecular stability towards electrons, exclusively using strong and stable acceptors is indeed viable. The Trz, cyano, and carbonyl undoubtedly guarantee high $\mathrm{BDE}_{\mathrm{f}}(-)$ values $(2.81-3.33 \mathrm{eV}$ for B4-B6, Figure $5 \mathrm{c})$. Nevertheless, abundant building blocks are always desired to meet the various needs in practical applications.

(a)<smiles></smiles>
$\mathrm{PhCz}(\mathrm{C} 1)$<smiles>CCCCC</smiles>
on 3-site: $\mathrm{C2}$ on 2-site: C5

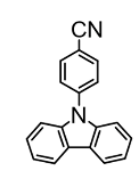

(b)

C8

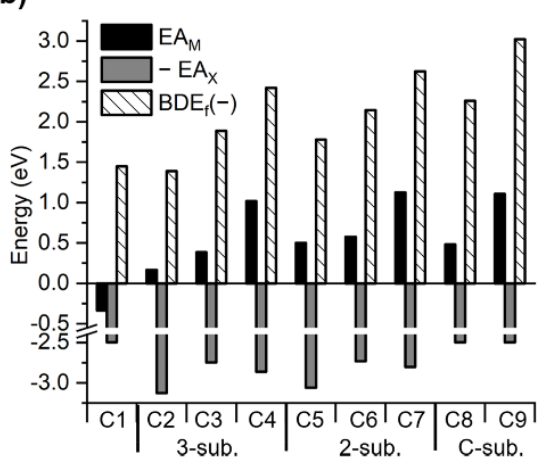

(c)
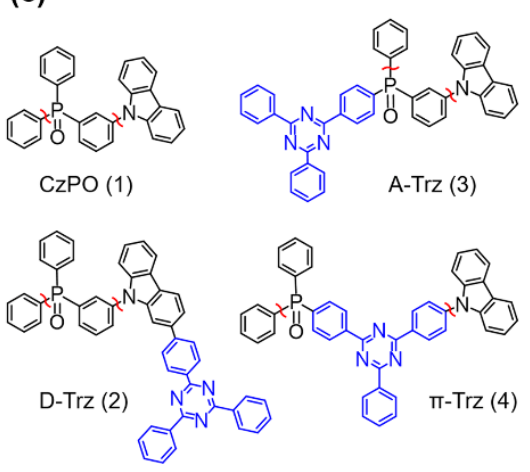

(d)

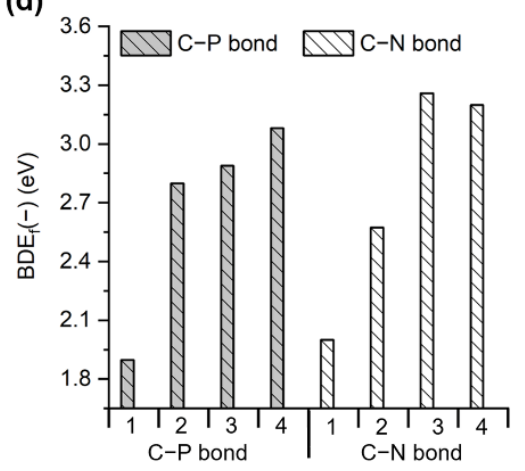

Figure 5. a) Chemical structures of $\mathrm{PhCz}$ and its derivatives, $\mathrm{BDEf}(-)$ values of the fragile $\mathrm{C}-\mathrm{N}$ bonds were calculated. $b) E A_{M}, E A x$, and $B D E_{f}(-)$ values of $P h C z-$ derivatives $\mathrm{C} 1-\mathrm{C}$. c) Chemical structures of $\mathrm{CzPO}$-derivatives with $\mathrm{D}-\mathrm{T}-\mathrm{A}$ backbones. The fragile $\mathrm{C}-\mathrm{N}$ and $\mathrm{C}-\mathrm{P}$ bonds are highlighted by the red markers. $\mathrm{d}$ ) $\mathrm{BDEf}(-)$ values of CzPO-derivatives. All calculations were at the M06-2X/def2-SVP level.

Here, we propose a superior strategy by introducing or constructing D-EWGs to make fragile bonds no longer fragile. The effectiveness can be evidenced by $\mathbf{C 1 - C 4}$, which retain the building blocks and fragile bonds in $\mathbf{A} \mathbf{1 - A 4}$, but combine them with extra strong EWGs, constructing D-EWGs at the center of the molecule. Markedly different from A1-A4, C1-C4 have substantially improved $\mathrm{BDE}_{\mathrm{f}}(-)$ values $(2.75-3.17 \mathrm{eV}$, Figure $5 \mathrm{~b})$. Meanwhile, these groups like PO could bring desirable optical and electrical performance, as evidenced by $\mathbf{C 4}$ with a tenfold increase of radiation rate constant and a fivefold decrease of non-radiation rate constants compared with PO-free counterparts, though the authors did not consider the stability issue. ${ }^{[11 a]}$ Our study for the first time disclosed the strategy can not only preserve the intrinsic advantages of the building blocks, but also address the intrinsic stability issues. The improved molecular stability of $\mathbf{C 1 - C 4}$ can be evidenced by the reported experiments. Lee et al. found that C1-based EOD are robust towards electrons. ${ }^{[13]}$ Kwon et al. replaced $\mathbf{A} 1$ with $\mathbf{C} 1$ as the host of a blue-OLED based on the consideration of BDE(-), which gave a $\sim 7$ fold increase of device lifetime. ${ }^{[6 \mathrm{k}]}$ Duan et al. found the C2-based OLEDs have a substantially prolonged lifetime compared with that based on $\mathbf{A 2},{ }^{[14]}$ in which the significantly increased $\mathrm{BDE}_{\mathrm{f}}(-)$ should not be neglected, although the authors did not mentioned this point. $\mathbf{C 3}$ is reported as a robust host even for pure blue phosphorescent OLEDs. ${ }^{[15]}$ The superior molecular stability of C4 to A4 has been demonstrated above. 
To enrich the building blocks for robust OLED materials, we designed three new blocks D1-D3 (Figure 5a) with D-EWG substructures. We expect they can stabilize various fragile bonds from electrons, and thus allowing high tunability for molecular design. Indeed, whether combined with $\mathrm{PO}$ or $\mathrm{Cz}$, the corresponding molecules all show high $\mathrm{BDE}_{\mathrm{f}}(-)$ values $(2.87-3.55 \mathrm{eV}$, Figure $5 \mathrm{~d})$. Finally, we show that the strategy is also effective for systems other than $\mathrm{Cz} / \mathrm{PO}$. For ETMs based on benzimidazole-derivatives, the prototypical TPBi (E1) has particularly low $\mathrm{BDE}_{\mathrm{f}}(-)(1.61 \mathrm{eV})$ for the $\mathrm{C}-\mathrm{N}$ bond. In comparison, E2 with extended m-conjugation has a higher $\mathrm{BDE}_{\mathrm{f}}(-)$ $(2.54 \mathrm{eV})$. Indeed, E2-based OLEDs have a $\sim 3$ fold increase of device lifetime compared with E1-based counterparts, ${ }^{[16]}$ in which the improved $\mathrm{BDE}_{\mathrm{f}}(-)$ should have a non-negligible contribution. Based on E2, we constructed 9,10-di(pyrimidin-2-yl)anthracene as a DEWG, which further improves $\mathrm{BDE}_{\mathrm{f}}(-)$ for $\mathbf{E 3}(2.92 \mathrm{eV}$, Figure $5 \mathrm{e})$. It is certain that the newly designed E3 with substantially higher $\mathrm{BDE}_{\mathrm{f}}(-)$ would be robust towards electrons. Overall, these examples further consolidate that our strategy would be general and effective for improving the molecular stability of various OLED materials.
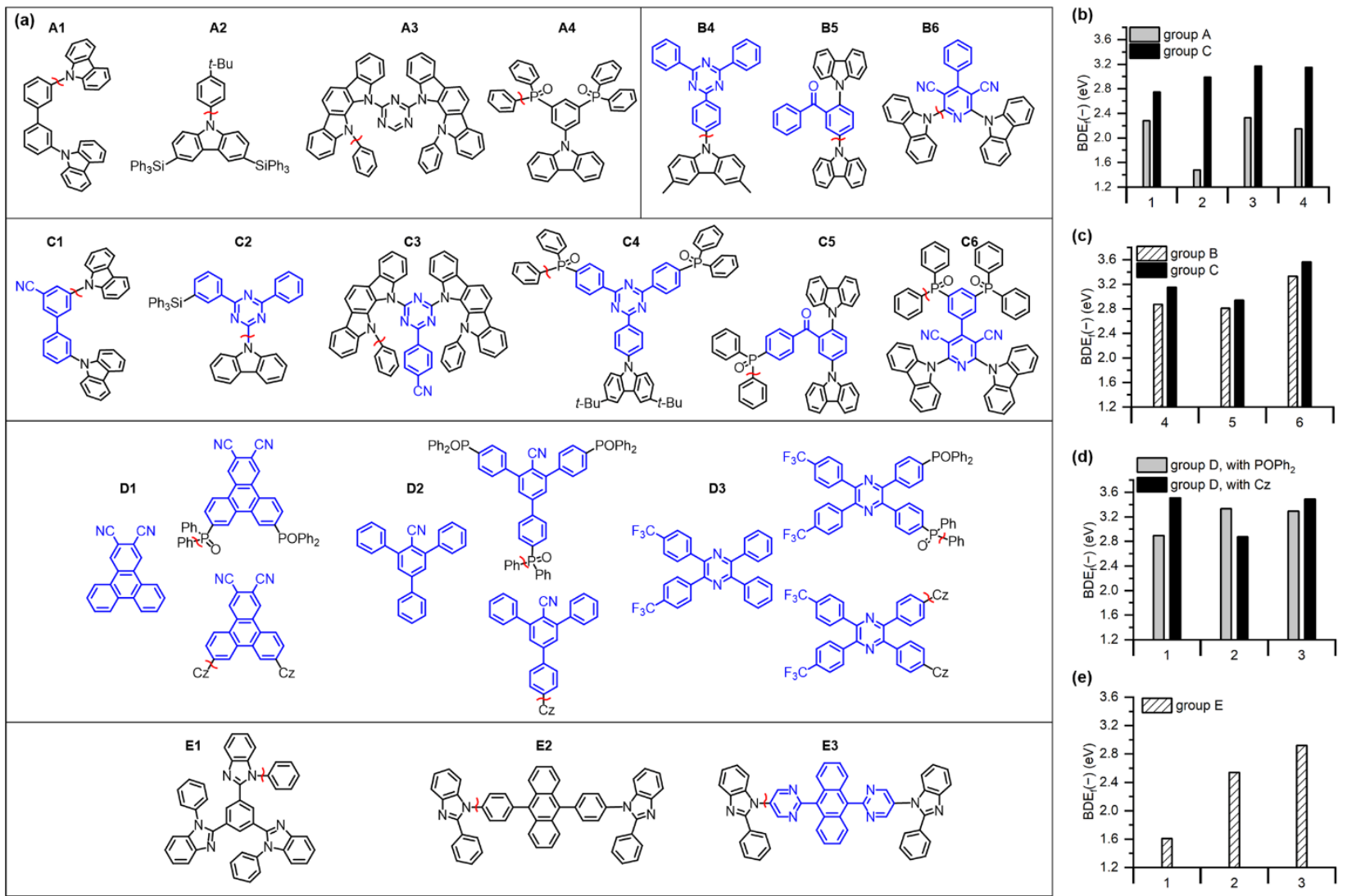

(e)

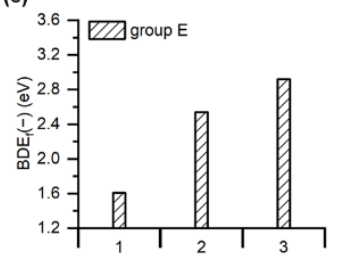

Figure 5. a) Chemical structures of the reported or newly designed OLED molecules. Their fragile bonds are highlighted by the red markers. $b-e), B D E_{f}(-)$ values of the molecules in: group A and $\mathrm{C} \mathrm{b}$ ); group B and $\mathrm{C} \mathrm{c)}$; group D d); and group E e). Among which, molecule C5, C6, E3, and all of the molecules in group D are newly designed in this work. The D-EWG moieties are highlighted in blue color. All calculations were at the M06-2X/def2-SVP level.

\section{Conclusion}

In this work, we first emphasized $\mathrm{BDE}_{\mathrm{f}}(-)$ is a less-studied molecular parameter but of vital importance for organic (opto)electronic materials, and confirmed the close relationship between $\operatorname{BDE}_{\mathrm{f}}(-)$, materials stability, and device lifetime. The comparative study on typical ETMs TP3PO and PO-T2T clearly demonstrated that active organic materials with lower $\operatorname{BDE}_{f}(-)$ do result in poorer materials stability and device lifetime for OLEDs. We then explored how to manipulate $\operatorname{BDE}_{f}(-)$. Based on the fundamental Hess's law, regulating $\operatorname{BDE}_{f}(-)$ is converted to the explicit modulations of $E A_{M}$ and $E A_{x}$. We found that introducing D-EWGs can significantly improve $E A_{M}$, but has much smaller effect on $E A_{x}$, thus substantially improving the $\mathrm{BDE}_{\mathrm{f}}(-)$ by $\sim 1 \mathrm{eV}$ for various OLED materials. Meanwhile, the bond cleavage process will be hindered by such $\mathrm{D}-$ EWGs due to their strong confinement of the negative charge. The effectiveness and generality of this strategy has been further validated in several newly designed molecules. Importantly, it provides a particular perspective to employ the originally vulnerable building blocks, which can make these groups no longer vulnerable towards electrons, thus largely enriches available building blocks for the rational design of robust OLED materials. At the end, it should be noted that this research looked into the manipulations of BDE(-) of C-X bonds, which helps to deepen the understanding on the relationship between molecular structures and $\operatorname{BDE}(-)$ values for all organic compounds. Hence, the strategy would be transferrable to other organic (opto)electronic materials. 


\section{Acknowledgements}

This work was supported by the National Key R\&D Program of China (No. 2016YFB0401003, 2016YFB0400702) and the National Science Fund of China (Grant Nos. 51525304) for financial support. We are grateful to Prof. Hui Xu et al. and Prof. Qisheng Zhang et al. for providing the sample of ptBCzPO2TPTZ and TCzTrz, respectively. We are also grateful to Prof. Jinpei Cheng and Dr. Jindong Yang for their valuable suggestions on our manuscript. We thank the Tsinghua National Laboratory for Information Science and Technology and Tsinghua Xuetang Talents Program for supporting the computational resources.

Keywords: anions $\bullet$ bond energy $\bullet$ chemical deterioration $\bullet$ intrinsic stability $\bullet$ organic light-emitting diodes

[1] a) O. Ostroverkhova, Chem. Rev. 2016, 116, 13279-13412; b) W. R. Mateker, M. D. McGee, Adv. Mater. 2017, 29, 1603940; c) J. T. E. Quinn, J. Zhu, X. Li, J. Wang, Y. Li, J. Mater. Chem. C, 2017, 5, 8654-8681. d) S. Scholz, D. Kondakov, B. Lüssem, K. Leo, Chem. Rev. 2015, 115, 8449-8503.

[2] a) C. Zhao, L., Duan, J. Mater. Chem. C, 2020,8, 803-820; b) W. Song, J. Y. Lee, Adv. Opt. Mater. 2017, 5, 1600901; c) S. Schmidbauer, A. Hohenleutner, B. König, Adv. Mater. 2013, 25, 2114-2129; d) S. K. Jeon, H. L. Lee, K. S., Yook, J. Y. Lee, Adv. Mater. 2019, 31, 1803524; e) X. Cai, S.-J. Su, Adv. Funct. Mater. 2018, 28, 1802558.

[3] a) N. C. Giebink, B. W. D'Andrade, M. S. Weaver, P. B. Mackenzie, J. J. Brown, M. E. Thompson, S. R. Forrest, J. Appl. Phys. 2008, 103, No. 044509; b) M. Shao, L. Yan, M. Li, I. Ilia, B. Hu, J. Mater. Chem. C 2013, 1, 1330-1336; c) Y. Zhang, J. Lee, S. R. Forrest, Nat. Commun. 2014, 5, 5008; d) W. Song, J. Y. Lee, T. Kim, Y. Lee, H. Jeong, Org. Electro. 2018, 57, 158-164; e) J. Sohn, D. Ko, H. Lee, J. Han, S.-D. Lee, C. Lee, Org. Electron. 2019, 71, 286-291; f) H. Xu, M. Wang, Z.-G. Yu, K. Wang, B. Hu, Adv. Phys. 2019, 68, 49-121; g) M. Tanaka, R. Nagata, H. Nakanotani, C. Adachi, Commun. Mater. $2020,1: 18$.

[4] a) D. Zhang, M. Cai, Y. Zhang, D. Zhang, L. Duan, Mater. Horiz. 2016, 3, 145-151; b) J. Lee, C. Jeong, T. Batagoda, C. Coburn, M. E. Thompson, S. R. Forrest, Nat. Common. 2017, 8:15566; c) H. Noda, H. Nakanotani, C. Adachi, Sci. Adv. 2018, 4: eaao6910; d) A. Ligthart, X. de Vruesm, L. Zhang, M. C. W. M. Pols, P. A. Bobbert, H. van Eersel, R. Coehoorn, Adv. Funct. Mater. 2018, 1804618; e) J. S. Bangsund, K. W. Hershey, J. R. Holmes, ACS Appl. Mater Interfaces 2018, 10, 5693-5699; f) D.-G. Ha, J. Tiepelt, M. A. Fusella, M. S. Weaver, J. J. Brown, M. Einzinger, M. C. Sherrott, T. V. Voorhis, N. J. Thompson, M. A. Baldo, Adv. Optical Mater. 2019, 1901048; g) S. H. Han, J. H. Jeong, J. W. Yoo, J. Y. Lee, J. Mater. Chem. C, 2019, 7, $3082-3089$.

[5] C. Jeong, C. Coburn, M. Idris, Y. Li, P. I. Djurovich, M. E. Thompson, S. R. Forrest, Org. Electron. 2019, 64, 15-21;

[6] a) D. Y. Kondakov, W. C. Lenhart, W. F. Nichols, J. Appl. Phys. 2007, 101, No. 024512; b) D. Y. Kondakov, J. Appl. Phys. 2008, 104, No. 084520; c) S. Scholz, K. Walzer, K. Leo, Adv. Funct. Mater. 2008, 18, 2541-2547; d) N. Lin, J. Qiao, L. Duan, H., Li, L. Wang, Y. Qiu, J. Phys. Chem. C 2012, 116, 1945119457; e) N. Lin, J. Qiao, L. Duan, L. Wang, Y. Qiu, J. Phys. Chem. C 2014, 118, 7569-7578; f) A. S. D. Sandanayaka, T. Matsushima, C. Adachi, J. Phys. Chem. C 2015, 119, 23845-23851; g) S. Y. Byeon, S. H. Han, J. Y. Lee, Adv. Optical Mater. 2017, 5, 1700387; h) A. Y. Freidzon, A. A. Safonov, A. A. Bagaturyants, D. N. Krasikov, B. V. Potapkin, A. A. Osipov, A. V. Yakubovich, O. Kwon, J. Phys. Chem. C 2017, 121, 22422-22433; i) R. Wang, Y.-L. Wang, N. Lin, R. Zhang, L. Duan, J. Qiao, Chem. Mater. 2018, 30, 8771-8781; j) M. Hong, M. K. Ravva, P. Winget, J.-L. Brédas, Chem. Mater. 2016, 28, 57915798. k) D. H. Ahn, J. H. Maneg, H. Lee, H. Yoo, R. Lampande, J. Y. Lee, J. H. Kwon, Adv. Optical Mater. 2020, 8, 2000102.

[7] Y. Luo, H., Aziz, G., Xu, Z. D. Popovic, Chem. Mater. 2007, 19, 2079-2083

[8] T. A. Schaub, E. M. Zolnhofer, D. P. Halter, T. E. Shubina, F. Hampel, K. Meyer, M. Kivala, Angew. Chem. 2016, 128, 13795-13799; T. A. Schaub, E. M. Zolnhofer, D. P. Halter, T. E. Shubina, F. Hampel, K. Meyer, M. Kivala, Angew. Chem. Int. Ed. 2016, 55, 13597-13601.

[9] a) C. S. Oh, J. M. Choi, J. Y. Lee, Adv. Optical Mater. 2016, 4, 1281-1287; b) J. Yoon, S. K. Kim, H. J. Kim, S. Choi, S. W. Jung, H. Lee, J. Y. Kim, D.-W. Yoon, C. W. Han, W.-S. Chae, J. H. Kwon, M. J. Cho, D. H. Choi, Chem. Eur. J. 2020. doi: 10.1002/chem.202002655; c) J.-Y. Kim, T.-R. Hong, I.-R. Choe, J.-A. Lee, H.-G. Ryu, B.-M. Seo, J.-H. Yang, C.-W. Han, J.-H. Baek, H.-C. Choi, I.-B. Kang, SID 2020 Digest 2020, ISSN 0097-996X/20/4803-0053.

[10] a) J. P. Cheng, Z. Zheng, Tetrahedron letters, 1996, 37, 1457-1460; b) A. Houmam, Chem. Rev. 2008, 108, 2180-2237; c) K. Daasbjerg, J. Chem. Soc. Perkin Trans. 1994, 2, 1275-1277.

[11] a) H. Yang, Q. Liang, C. Han, J. Zhang, H. Xu, Adv. Mater. 2017, 29, 1700553; b) S. Y. Lee, C. Adachi, T. Yasuda, Adv. Mater. 2016, 28, 4626-4631; c) C. Li, C. Duan, C. Han, H. Xu, Adv. Mater. 2018, 30, 1804228; d) S. Gong, Y.-L. Chang, K. Wu, R. White, Z.-H. Lu, D. Song, C. Yang, Chem. Mater. 2014, 26 1463-1470; e) J. Jia, L. Zhu, Y. Wei, Z. Wu, H. Xu, D. Ding, R. Chen, D. Ma, W. Huang, J. Mater. Chem. C, 2015, 3, 4890-4902.

[12] Z. Liu, F. Cao, T. Tsuboi, Y. Yue, C. Deng, X. Ni, W. Sun, Q. Zhang, J. Mater. Chem. C, 2018, 6, 7728-7733.

[13] S.-G. Inn, N. Lee, S. O. Jeon, M. Sim, H. Kang, Y. Jung, D. H. Huh, Y. M. Song, S. Y. Lee, M. Numata, H. Miyazaki, R. Gómez-Bombarelli, J. AguileraIparraguirre, T. Hirzel, A. Aspuru-Guzik, S. Kim, S. Lee, Adv. Sci. 2017, 4, 1600502.

[14] D. Zhang, L. Duan, Y. Li, H. Li, Z. Bin, D. Zhang, J. Qiao, G. Dong, L. Wang, Y. Qiu, Adv. Funct. Mater. 2014, 24, 3551-3561.

[15] M. Jung, M. H. Lee, H. Y. Lee, T. Kim, Mater. Horiz., 2020, 7, 559-565.

[16] D. Zhang, P. Wei, D. Zhang, L. Duan, ACS Appl. Mater. Interface 2017, 9, 19040-19047. 